\title{
Parrondo's games and the zipping algorithm
}

\author{
Pau Amengual and Raúl Toral \\ Instituto Mediterráneo de Estudios Avanzados, IMEDEA (CSIC-UIB), \\ ed. Mateu Orfila, Campus UIB, E-07122 Palma de Mallorca, Spain
}

\begin{abstract}
We study the relation between the discrete-time version of the flashing ratchet known as Parrondo's games and a compression technique used very recently with thermal ratchets for evaluating the transfer of information negentropy - between the Brownian particle and the source of fluctuations. We present some results concerning different versions of Parrondo's games showing, for each case, a good qualitative agreement between the gain and the inverse of the entropy.
\end{abstract}

Keywords: Ratchets, Parrondo's paradox, information theory

\section{INTRODUCTION}

In recent years, the microscopic field of Brownian particles has received much attention due to new transport phenomena under certain conditions. The main features involve breaking of spatial inversion symmetry and a thermal bath that drives the system out of equilibrium ${ }^{1}$ in order to obtain directed transport. The former condition is accomplished through an asymmetric potential, usually a ratchet-like potential. The latter can be achieved in different ways, either by a periodic or stochastic forcing - what it is known as pulsating ratchets, or by an additive driving force unbiased on average - known as tilting ratchets. The flashing ratchet model corresponds to the class of pulsating ratchets and it consists on switching on and off either periodically or stochastically a ratchet potential. This model has been used recently for DNA transport ${ }^{2}$ and separation of biological macromolecules. ${ }^{3}$

Recently, Arizmendi et. $a l^{4}$ have quantified the transfer of information - negentropy - between the Brownian particle and the nonequilibrium source of fluctuations acting on it. They coded the particle motion into a binary string of 0's and 1's, and then zipped the resulting binary file using the Lempel and Ziv algorithm ${ }^{5}-$ available $^{2}$ through the gzip program. In this way they obtained an estimation of the entropy per character $h$, as the ratio between the length of the zipped file and the original length file, when the text length tends to infinity. They used this method to estimate the entropy per character of the ergodic source for different values of the flipping rate, with the result that there exists a close relation between the current in the ratchet and the net transfer of information in the system.

The aim of this paper is to apply this technique to a discrete-time and space version of the Brownian ratchet, known in the literature as Parrondo's paradox.

Parrondo's paradox ${ }^{6-12}$ is based on a combination of two negatively biased games - losing games - which when combined give rise to a positively biased game - a winning game. This paradox appeared as a translation of the physical model of the Brownian ratchet into game-theoretic terms. But although it was inspired by the flashing ratchet model, there has been no quantitative demonstration of their relation until very recently. ${ }^{13-15}$

More precisely, the so called Parrondo's paradox is based on the combination of two games (Parrondo's games). One of them, say game A, is a simple coin tossing game where the player has a probability $p$ of winning one unit of capital and a probability $1-p$ of losing one unit of capital. The second game, game B, is a capital dependent game, where the probability of winning depends on the capital of the player modulo a given number

emails:pau@imedea.uib.es, raul@imedea.uib.es; http://www.imedea.uib.es/PhysDept

Noise in Complex Systems and Stochastic Dynamics II, edited by Zoltán Gingl,

José M. Sancho, Lutz Schimansky-Geier, Janos Kertesz, Proc. of SPIE Vol. 5471 
$M$. For the original games, $M$ is set to three and there are only two possible values for the winning probabilities, $p_{0}$ when the capital is a multiple of three and $p_{1}$ otherwise. Their numerical values are

$$
\left\{\begin{array}{c}
p=\frac{1}{2}-\epsilon, \\
p_{0}=\frac{1}{10}-\epsilon, \\
p_{1}=\frac{3}{4}-\epsilon,
\end{array}\right.
$$

here $\epsilon$ is nothing but a biasing parameter that converts games A and B into losing games. When $\epsilon=0$ it can be demonstrated that the fairness condition is fulfilled for both games, that is $\prod_{j=0}^{M-1} p_{i}=\prod_{j=0}^{M-1}\left(1-p_{i}\right)$. As soon as $\epsilon>0$ the latter condition no longer applies and $\mathrm{A}$ and $\mathrm{B}$ are both losing games.

The combination game, game $\mathrm{AB}$, is obtained mixing game $\mathrm{A}$ and game $\mathrm{B}$ with probability $\gamma$. As a result of this combination we obtain a winning game, even when $\epsilon$ is equal to zero. The corresponding winning probabilities are given by the following expressions

$$
\left\{\begin{array}{l}
p_{0}^{\prime}=\gamma p+(1-\gamma) p_{0} \\
p_{1}^{\prime}=\gamma p+(1-\gamma) p_{1}
\end{array}\right.
$$

Several other versions of the games have been introduced. In the so-called cooperative games, ${ }^{16,17}$ one considers an ensemble of interacting players. In the history dependent games, ${ }^{18,19}$ the probabilities of winning depend on the history of previous results of winnings and losing. Finally, in the games with self-transition, ${ }^{20}$ there is a non-zero probability that the capital can stay the same (not winning or losing) in a given toss of the coins.

Some previous works in the literature has related Parrondo's games and information theory. Pearce, in Ref., ${ }^{21}$ considers the relation between the entropy and the fairness of the games, and the region of the parameter space where the entropy of game $\mathrm{A}$ is greater than that of $\mathrm{B}$ and $\mathrm{AB}$. Harmer et. $a l^{22}$ studied the relation between the fairness of games A and B and the entropy rates considering two approaches. The first one consisted on calculating the entropy rates not taking into account the correlations present on game B, finding a good agreement between the region of maximum entropy rates and the region of fairness. In the second approach they introduced these correlations obtaining lower entropy rates and no significant relation between fairness and entropy rates for game B.

In this paper we aim to relate the current or gain in Parrondo's games with the variation of information entropy of the binary file generated. Following the same approach as in Ref., ${ }^{4}$ we have carried out simulations for different versions of Parrondo's games, including the original version, the history dependent games, Parrondo's games with self-transition and cooperative games mentioned above.

\section{SIMULATION RESULTS}

We have carried out numerical simulations of the different versions of the games. In the simulations, the evolution of the capital of the player has been converted to a binary string of 0's and 1's, corresponding to a decrease and an increase of capital respectively. We must remark that each one of these characters is stored as a byte (eight bits). A binary file has then been created for different values of the $\gamma$ parameter. In order to obtain the desired effect there must be a certain delay time, which we will denote by $\delta_{t}$, between measurements of the capital of the player.

The binary files created have been zipped using the gzip (v. 1.3) program, to obtain an estimation of the entropy per character $h$. For a better comparison with the current - or gain - generated when alternating between games $\mathrm{A}$ and $\mathrm{B}$, we will define $R=\frac{1}{h}$, accounting, in some sense, for the known information about the system. In the Appendix there is an extract of the $\mathrm{C}$ program used for the case of the original Parrondo's games.

In Fig. 1 we compare the gain when alternating between games A and B with probability $\gamma$ with the value of $R$ obtained for different values of the $\delta_{t}$ parameter. The numerical curves are shifted to the origin, that is, we plot $\Delta R=R_{\gamma}-R_{\gamma=0}$.

We find a good qualitative agreement between the increase in the gain and the decrease (increase) in entropy $(R)$ as the $\gamma$ parameter is varied. Although both curves do not fully coincide, the important fact to consider here 


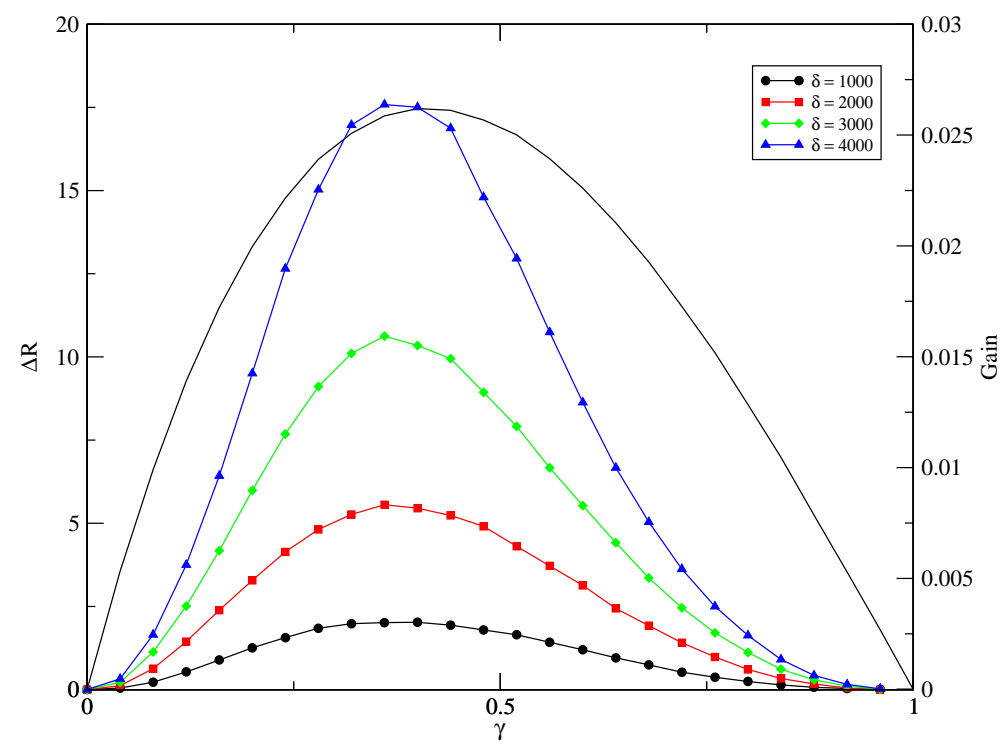

Figure 1. Comparison of the gain in the original Parrondo's games with $\Delta R$ calculated for different $\delta_{t}$ and $\gamma$ values. The probabilities for the Parrondo game are $p=\frac{1}{2}, p_{0}=\frac{1}{10}$ and $p_{1}=\frac{3}{4}$. The values used are $\delta_{t}=1000$ (circles), 2000 (squares), 3000 (diamonds) and 4000 (triangles).

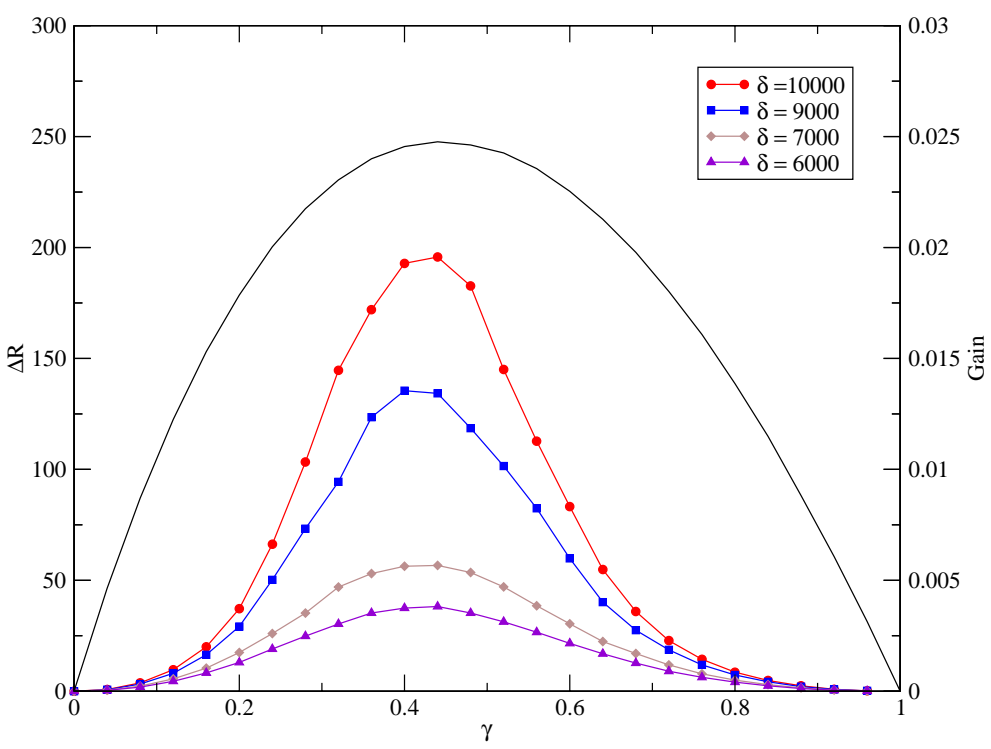

Figure 2. Comparison between the inverse of the entropy per character $R$, calculated for different $\delta_{t}$ values, and the gain for the Parrondo's games $A$ and $B$ with self-transition varying $\gamma$. The values for the probabilities are: $p=\frac{9}{20}, r=\frac{1}{10}$, $p_{0}=\frac{3}{25}, r_{0}=\frac{2}{5}, p_{1}=\frac{3}{5}$ and $r_{1}=\frac{1}{10}$. See Ref. ${ }^{20}$ for an explanation of these parameters. The values used are $\delta_{t}=6000$ (triangles), 7000 (diamonds), 9000 (squares) and 10000 (circles). 


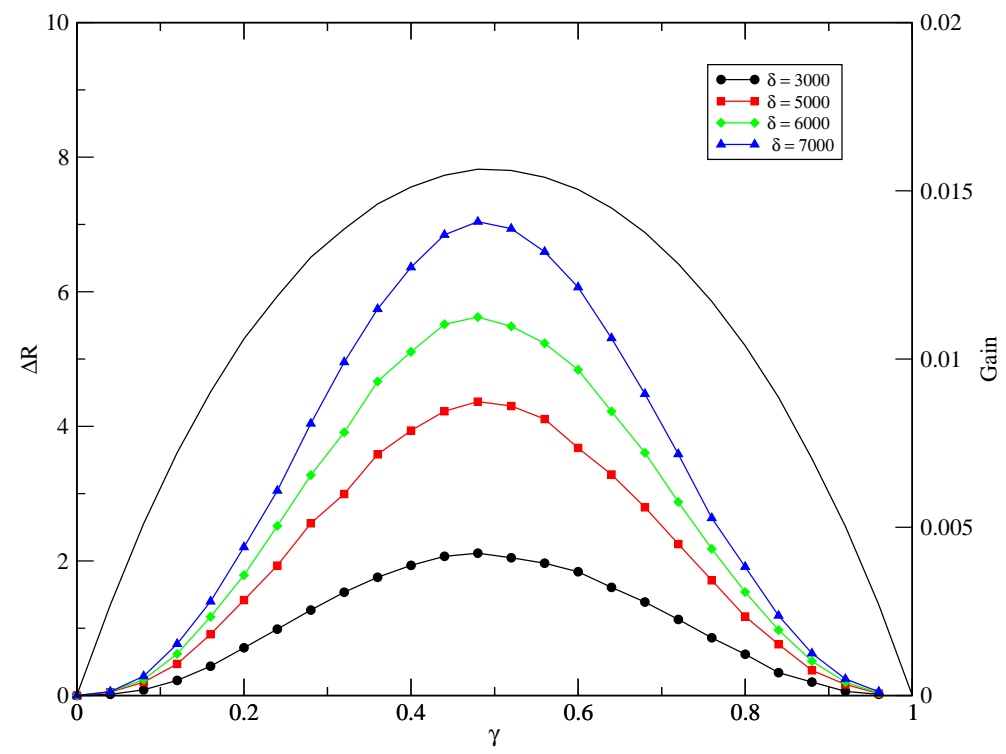

Figure 3. Curve for the inverse of the entropy per character $R$ and the gain for the history dependent games, alternating between two games with probabilities: $p_{1}=\frac{9}{10}, p_{2}=p_{3}=\frac{1}{4}, p_{4}=\frac{7}{10} ; q_{1}=\frac{2}{5}, q_{2}=q_{3}=\frac{3}{5}$ and $q_{4}=\frac{2}{5}$. See Ref. ${ }^{18}$ for an explanation of these parameters. The values for the $\delta_{t}$ are: 3000 (circles), 5000 (squares), 6000 (diamonds) and 7000 (triangles).

is that the $\gamma$ value for which there is the maximum decrease in entropy agrees with the value for the maximum gain in the games. This decrease in the entropy of the system implies that there exists an increase in the amount of information that we know about the system.

In Fig. 2 we compare the gain curve and the entropy curve for the case of Parrondo's games with selftransition. Again in this case the maximum gain coincides with the $\gamma$ value for the minimum entropy per character for all values of $\delta_{t}$.

Finally, in Figs. 3 and 4 we show the comparison in the case of the history dependent games, and cooperative games, sharing all them the same features as the previous games analyzed before.

\section{THEORETICAL ANALYSIS}

In this section we develop a simple argument that explains the observed relation between the gain of the games and their entropy. In the seminal work by Shannon ${ }^{23}$ the entropy per character of a text produced by an ergodic source is given by the following expression

$$
H=-\sum_{i} p_{i} \cdot \log \left(p_{i}\right)
$$

where $p_{i}$ denotes the probability that the source will 'emit' a given symbol $a_{i}$, and the sum is taken over all possible symbols that the source can emit.

If the source can be found in several states, each one with probability $P_{j}$, then the entropy reads

$$
H=\sum_{j=1}^{M} P_{j} H_{j}=-\sum_{j, i} P_{j} p_{i}^{j} \log \left(p_{i}^{j}\right)
$$

In the last equation $p_{i}^{j}$ denotes the probability of emitting the symbol $a_{i}$ when the source is found in the state $j$. 


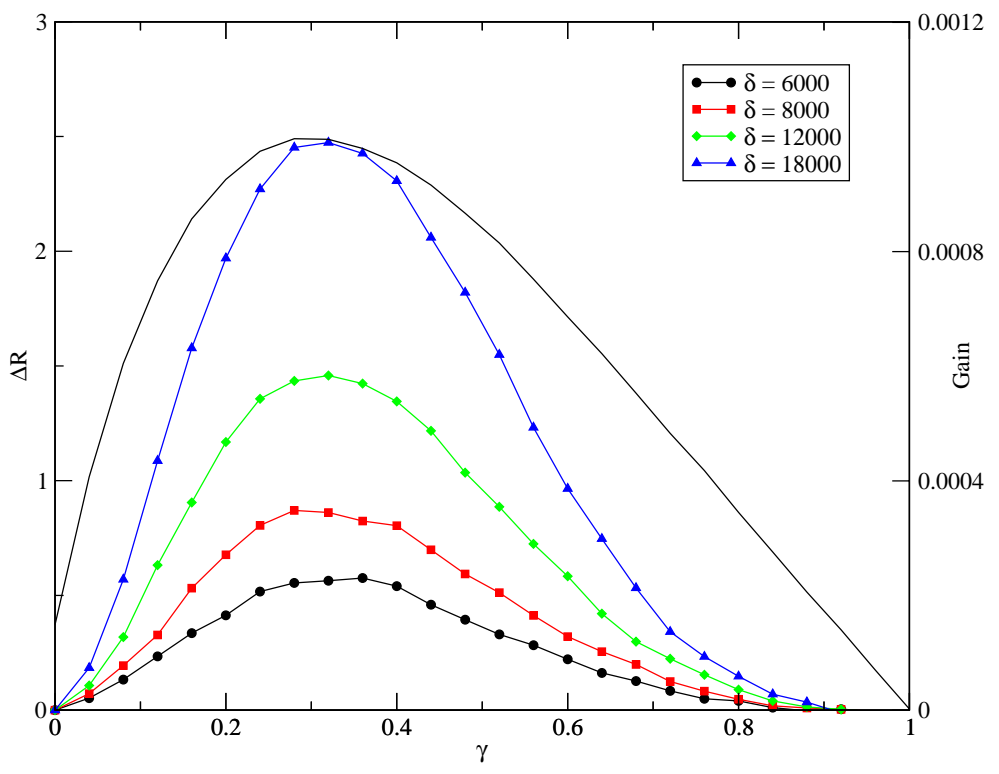

Figure 4. Curve for the inverse of the entropy per character $R$ and the gain for the cooperative version with probabilities: $p=\frac{1}{2}, p_{1}=1, p_{2}=p_{3}=\frac{16}{100}, p_{4}=\frac{7}{10}$ and $N=150$ players for different values of the $\gamma$ parameter. See Ref. ${ }^{16}$ for an explanation of these parameters. The values of the $\delta_{t}$ are: 6000 (circles), 8000 (squares), 12000 (diamonds) and 18000 (triangles).

Remember that we measure and store the capital of the player after $\delta_{t}$ rounds have been played. When $\delta_{t}$ equals one it means that we measure the capital of the player in each round. Translating this into entropic terms we have that, considering the original Parrondo's games, our source will be in two possible states, say, when the capital is multiple of three and otherwise. From Markov chain theory ${ }^{24}$ we know that the stationary probabilities of finding the capital in each of the states are given by

$$
\begin{gathered}
\Pi_{0}=\frac{1}{D}\left(1-p_{1}+p_{1}^{2}\right) \\
\Pi_{1}=1-\Pi_{0}=\frac{1}{D}\left(2-p_{0}-p_{1}+2 p_{0} p_{1}\right)
\end{gathered}
$$

where $D=3-p_{0}-2 p_{1}+2 p_{0} p_{1}+p_{1}^{2}$.

It is interesting to consider our source (the capital of the player) as composed of several sources, each one with an homogeneous statistical structure, i.e. they are ergodic sources. Each source then will have a determined probability of 'emitting' a zero or a one, as the player has a different winning probability depending on whether his capital is multiple of three or not. If we consider that the source will emit the symbol 1 with probability $p_{i}$ and 0 with probability $1-p_{i}$ then Eq. 4 reads

$$
\begin{aligned}
H & =\sum_{j=1}^{M} \Pi_{j} H_{j}=-\sum_{j, i} \Pi_{j} p_{i}^{j} \log \left(p_{i}^{j}\right) \\
& =-\Pi_{0} \cdot\left(p_{1} \cdot \log \left(p_{1}\right)+\left(1-p_{1}\right) \cdot \log \left(1-p_{1}\right)\right)-\left(1-\Pi_{0}\right) \cdot\left(p_{2} \cdot \log \left(p_{2}\right)+\left(1-p_{2}\right) \cdot \log \left(1-p_{2}\right)\right)
\end{aligned}
$$

In Fig. 5 we plot the inverse of Eq. 6 for the values of $p_{i}^{j}$ and $\Pi_{i}$ corresponding to the original Parrondo's games together with the numerical curve obtained when zipping the files obtained with $\delta_{t}=1$. It is worth noting how the negentropy decreases as $\gamma$ increases, translating into an increase of known information about the system.

Alternatively, we can now estimate how the entropy changes for $\delta_{t} \gg 1$, when we allow a large amount of time between measures. As $\delta_{t}$ is increased, the statistical description used above cannot be used anymore. For higher $\delta_{t}$ values, the system gradually loses its memory about its previous state and so what we obtain are measures 


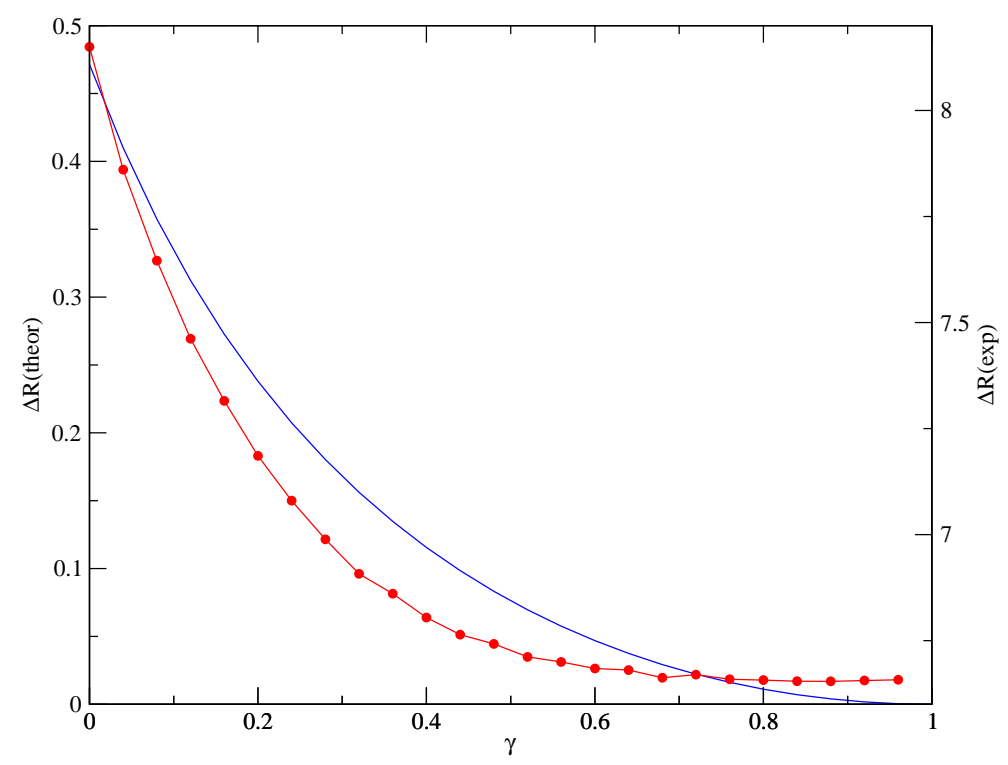

Figure 5. Plot of the theoretical curve for the inverse of the entropy per character and the numerical curve (circles) for the case when $\delta_{t}=1$ step.

that can be considered as statistically independent, as we will not know for sure which will be the probability of finding the source in any of the two possible states. Now the dominant term will be the averaged probability - over all possible states- of finding the capital of the player with a value higher than the previous measure.

Considering the same stationary probabilities $\Pi_{0}$ and $\Pi_{1}$ given by Eq. 5 we can obtain the expressions for the average winning and losing probabilities over these two states as

$$
\begin{aligned}
& p_{\text {win }}=\sum_{j=1}^{M} \Pi_{j} p_{j}^{i}=\Pi_{0} p_{0}^{\prime}+\Pi_{1} p_{1}^{\prime} \\
& p_{\text {lose }}=\Pi_{0} \cdot\left(1-p_{0}^{\prime}\right)+\Pi_{1} \cdot\left(1-p_{1}^{\prime}\right) .
\end{aligned}
$$

It can be noticed in all Figs. 1,2,3,4 that as $\delta_{t}$ increases, the entropy curve acquires a more pronounced peak. This effect can be explained due to the fact that as the time between measures increases, the probability of finding the player with a higher (lower) capital increases (decreases). We can give an estimate of the probability that after $\delta_{t}$ rounds played, the capital of the player will be greater $\left(p_{>}\right)$than it was initially by considering the probability that there have been less that $\delta_{t} / 2$ loses in the $\delta_{t}$ coin tosses,

$$
p_{>}=\sum_{k=0}^{\frac{\delta}{2}}\left(\begin{array}{c}
\delta_{t} \\
k
\end{array}\right) \cdot p_{\text {win }}^{\delta_{t}-k} \cdot p_{\text {lose }}^{k}
$$

These previous equations together with

$$
H=-p_{>} \cdot \log \left(p_{>}\right)-\left(1-p_{>}\right) \cdot \log \left(1-p_{>}\right),
$$

allows one to obtain the entropy per character. In Fig. 6 we can see the curve for the variation of the inverse of information entropy for different values $\delta_{t}$. In this case the negentropy decreases as $\delta_{t}$ increases, reflecting an increase of known information about the system.

This theoretical explanation also holds for the case of history dependent Parrondo's games and Parrondo's games with self-transition. For the latter case, though, we must take into account the fact that there exists a non negligible possibility that the capital can remain the same after a round played. In this case we need only 


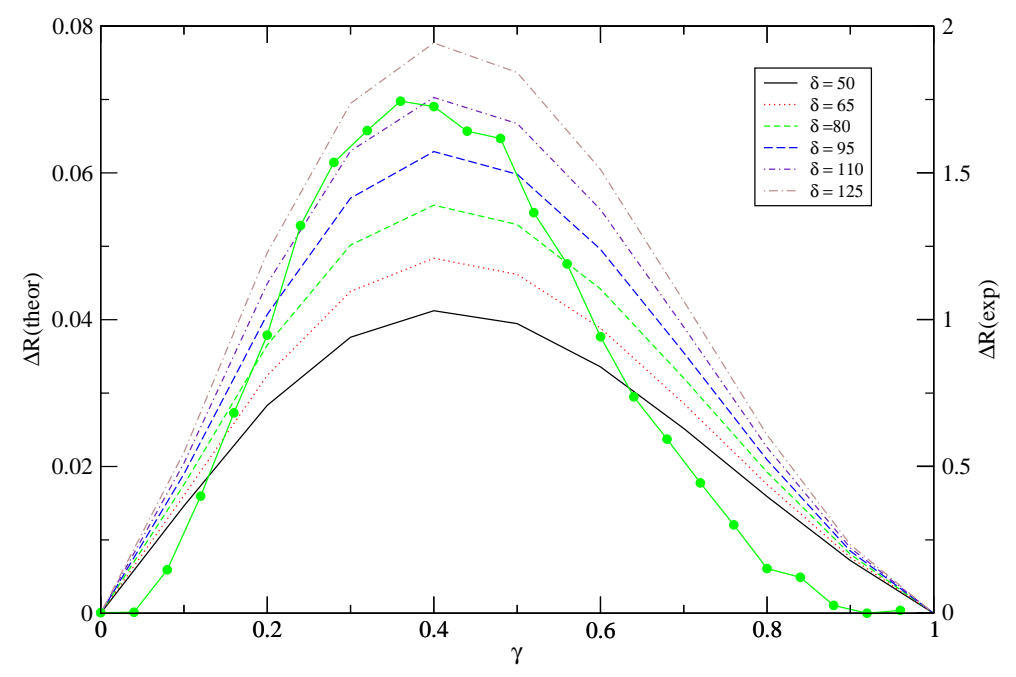

Figure 6. Plot of the theoretical curves for the inverse of the entropy per character for different values of $\delta_{t}$, together with the numerical curve obtained for $\delta_{t}=500$ steps.

to consider a source that will emit three different symbols, depending on whether the capital is greater, equal or smaller than our previous measure.

As a conclusion, we have demonstrated that there exists a transfer of information in the case of Parrondo's games, considered as a discrete-time and space version of the flashing ratchet. This effect takes place in every existing version of the games analyzed, showing the robustness of it. The qualitative analysis for the case of the original Parrondo's games have demonstrated the fact that the capital of the player can be thought of as a mixed source, composed of two ergodic sources, depending on whether the capital is a multiple of three or not.

We have also given some insight about the behavior of the information entropy for low and high values of $\delta_{t}$, and why there is a decrease (increase) in the entropy per character $h$ (information) as $\delta_{t}$ increases.

\section{Acknowledgments}

We thank C. M. Arizmendi for several discussions, including the sharing of useful information about the method. We acknowledge financial support from MCYT (Spain) and FEDER through projects BFM2000-1108 and BFM2001-0341-C02-01. P.A. is supported by a grant from the Govern Balear.

\section{APPENDIX}

We provide the $\mathrm{C}$ program used for creating the binary files that will be zipped for obtaining the entropy per character $h$.

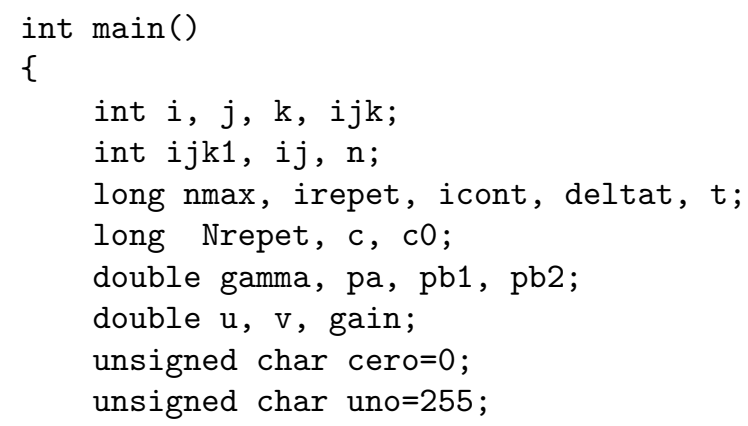


srand48(342342);

FILE *current;

current = fopen ("gain.dat", "w");

/* We fix the lenght in bytes of the binary file */

Nrepet $=300000$;

/* We impose the delay time between measurements */

deltat $=500$;

/* Probabilities of the original Parrondo's games */

$\mathrm{pa}=1 . / 2 . ;$

$\mathrm{pb} 1=1 . / 10 . ;$

$\mathrm{pb} 2=3 . / 4$;

$i j k=0$;

for $($ gamma $=0 . ;$ gamma $<=1.0 ;$ gamma $=$ gamma +0.04$)\{$

/* we create a binary file for each value of gamma */

sprintf (q, "gamma\%d.dat", ijk);

FILE *data;

data $=$ fopen $(\mathrm{q}, " \mathrm{w} ")$;

$++i j k$;

$\mathrm{cO}=0$

$c=0$;

$i=0$;

$i j k 1=0$

$t=0$;

while (i<Nrepet) \{

$++\mathrm{t}$

if $($ drand48( $)<$ gamma $)\{$

/* we play game A */

if $(\mathrm{drand} 48()<\mathrm{pa})\{$

$++c ;\}$

else \{

- c ;

\}

$++i j k 1 ;\}$

else\{ 


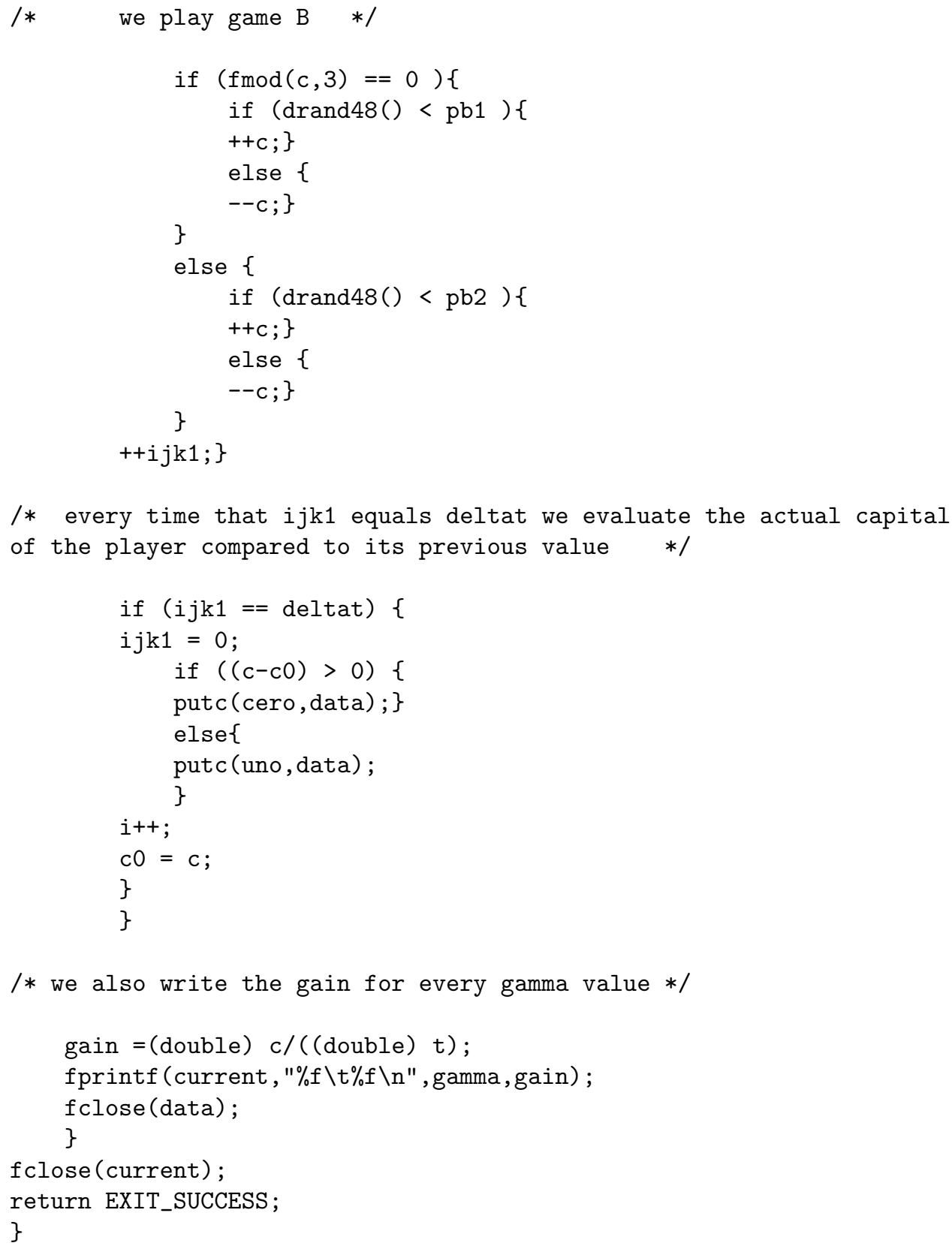

\section{REFERENCES}

1. Reimann, P. 2002 Brownian motors: noisy transport far from equilibrium. Physics Reports 361, 57-265.

2. Bader, J. S., Hammond, R. W., Henck, S. A., Deem, M. W., McDermott, G. A., Bustillo, J. M., Simpson, J. W., Mulhern, G. T. \& Rothberg, J. M. 1999 DNA transport by a micromachined Brownian ratchet device. Proc. Nat. Acad. Sciences 96, 13165-13169.

3. Duke, T. A. J. \& Austin, R. H. 1998 Microfabricated Sieve for the Continuous Sorting of Macromolecules. Physical Review Letters 80, 1552-1555.

4. Arizmendi, C. M., Sanchez, J. R. \& Family, F. 2003 Zipping thermal ratchets. Physica A 327, 111-114.

5. Lempel, A. \& Ziv, J. 1977 IEEE Trans. Inform. Theory 23, 337. 
6. Parrondo, J. M. R. 1996 How to cheat a bad mathematician. EEC HCEM Network on Complexity and Chaos (\#ERBCHRX-CT 940 546), ISI, Torino, Italy. Unpublished.

7. Harmer, G. P., \& Abbott, D. 1999a Parrondo's paradox. Statistical Science 14, 206-213.

8. Harmer, G. P., \& Abbott, D. 1999b Losing strategies can win by Parrondo's paradox. Nature 402, 864.

9. Harmer, G. P., Abbott, D., \& Taylor, P. G. 2000 The Paradox of Parrondo's Games. Proc. Roy. Soc. London A 456, 247-259.

10. Harmer, G. P., Abbott, D., \& Taylor, P. G., Parrondo, J. M. R. 2001 Brownian ratchets and Parrondo's games. Chaos 11, 705-714.

11. C. Van den Broeck, P. Reimann, R. Kawai, P. Hänggi, Lect. Notes in Physics 527 (1999) 93.

12. Harmer, G. P. \& Abbott, D. 2002 A review of Parrondo's paradox. Fluctuation and Noise Letters 2, R71R107.

13. Toral, R., Amengual, P. \& Mangioni, S. 2003 Parrondo's games as a discrete ratchet. Physica A 327, $105-110$.

14. Toral, R., Amengual, P. \& Mangioni, S. 2003, A Fokker-Planck description for Parrondo's games. Proc. SPIE Noise in complex systems and stochastic dynamics (eds. L. Schimansky-Geier, D. Abbott, A. Neiman \& C. Van den Broeck), 5114, 309-317. Santa Fe.

15. Allison, A., \& Abbott, D. 2002 The physical basis for Parrondo's games. Fluctuation and Noise Letters 2, L327-L342.

16. Toral, R. 2001 Cooperative Parrondo's games. Fluctuations and Noise Letters 1, L7-L12.

17. Toral, R. 2002 Capital redistribution brings wealth by Parrondo's paradox, Fluctuationsand Noise Letters 2, L305.

18. Parrondo, J. M. R., Harmer, G. P. \& Abbott, D. 2000 New paradoxical games based on Brownian ratchets. Physical Review Letters 85, 5226-5229.

19. Kay, R. J. \& Johnson, N. F. 2002 Winning combinations of history-dependent games. Physical Review E 67, 056128(6).

20. Amengual, P., Allison, A., Toral, R. \& Abbott, D. 2003 Discrete-time ratchets, the Fokker-Planck equation and Parrondo's paradox. To appear in Proc. Roy. Soc. London A.

21. Pearce, C. E. M. 2000 Entropy, Markov Information Sources and Parrondo Games. Unsolved Problems of Noise and Fluctuations (eds. D. Abbott \& L. B. Kish), 511, 207-212. American Institute of Physics (AIP).

22. Harmer, G. P., Abbott, D., Taylor, P. G., Pearce, C. E. M. \& Parrondo, J. M. R. 2000 Information Entropy and Parrondo's Discrete-Time Ratchet. Stochastic and Chaotic Dynamics in the Lakes: STOCHAOS (eds. D. S. Broomhead, E. A. Luchinskaya, P. V. E. McClintock \& T. Mullin), 502, 544-549. American Institute of Physics (AIP).

23. Shannon, C. E. 1948 A Mathematical Theory of Communication. The Bell System Technical Journal 27, 379-423, 623-656.

24. Karlin, S. \& Taylor, H. M. 1975 A first course in Stochastic Processes. Academic Press. New York. 